\section{Quality in Nuclear Medicine}

A.W.J.M. Glaudemans, J. Medema, A.K. van Zanten, and R.A.J.O. Dierckx, Eds., C.T.B. (Kees) Ahaus, Guest Ed.

Cham, Switzerland: Springer International Publishing, 2017, 466 pages, $\$ 135.99$

This textbook is a comprehensive overview of policies and procedures in nuclear medicine as well as a discussion of its scientific successes. Historically, nuclear medicine emerged when Henri Becquerel and Marie Curie discovered radioactive uranium and its high energy rays. Over the past century, nuclear medicine radiopharmaceuticals and diagnostic tests developed, leading to the introduction of high-tech equipment, robotics, and process technology. As the nuclear medicine industry emerged, laws, standards, and guidelines led to structured quality controls encompassing all its processes. Quality in Nuclear Medicine aims to provide the reader with an integrated approach to quality and quality management aspects in nuclear medicine.

One interesting chapter discusses teamwork, leadership, and management dynamics as a way to create a culture of continuous improvement. Using an evidence-based and modular teamwork system deployed in concordance with a novel medical leadership development program, a practical approach can be embraced, improving performance and patient safety within nuclear medicine departments that can also be applied across multiple disciplines. Evidence-based teamwork can be summarized by a few groundbreaking studies concerning the malignant "blame culture" associated with organizations that rely predominantly on a hierarchical, compliance-based functional management system. Medical organizations generally fall under the latter type organizational system, often lagging behind the current mainstem business models in terms of teamwork and leadership. The book shines light on this looming discrepancy. This is an example of the many refreshingly progressive themes throughout Quality in Nuclear Medicine.

One of the problems with nuclear medicine has been dealing with the decommissioning of cyclotron facilities used in the production of radionuclides and the disposal of radioactive material. In the past, many accelerators were installed mainly for nuclear medicine research and teaching at universities, with most of them currently having been dismantled. When PET techniques became popular beginning in the 1980s, cyclotron facilities were installed in both research facilities and large community hospitals. With the aging of many of these facilities, proper decommissioning becomes important, especially since a second-hand market is not available for the equipment. New techniques for decommissioning and new regulations were put in place to deal with the radioactive material, dismantle the equipment, and contain the concrete vaults. The authors provide an interesting explanation of the steps involved in this process.

Another thought-provoking chapter is the final chapter on the future of nuclear medicine. Nuclear medicine established itself as an independent specialty in several European Union countries since the 1970s; however, despite this, many countries have been slow to embrace nuclear medicine as a recognized independent specialty. The authors dive into this fascinating conundrum by highlighting the evolution of nuclear medicine training and abolishing some misconceptions about the field. In recent years, there has been a refocus on personalized medicine and multidisciplinary care to deliver the best treatment to patients. As competition and "turf battles" between specialties increase, the authors emphasize how nuclear medicine trainees should spend more time acquiring sound knowledge of the affiliated specialties. This would ensure comprehension of the needs of the various referrers and help establish the value of nuclear medicine as a specialty. Although nuclear medicine has been cast as the scapegoat when it comes to the harm elicited to patients through radiation by means of medical imaging, this chapter works to inform the reader on the facts associated with radiation exposure and dispel the myths surrounding the specialty of nuclear medicine. The authors question the current status quo and demand from its leaders in the field to come forth and advocate for their specialty. In this current era of "individualized medicine," nuclear medicine specialists must adapt to this model to ensure they become a core member of the medical team.

Quality in Nuclear Medicine is a multiauthor textbook written by experts in the respective fields. The book discusses a wide range of global subjects in nuclear medicine including laws and standards, radiation safety, nuclear accidents, medical devices and equipment, preclinical quality issues, leadership, and the role of international organizations. Readers will find the book to be an excellent wealth of knowledge and guidance in the different aspects of quality in nuclear medicine.

\author{
Joseph Stein \\ Stony Brook University Hospital \\ Stony Brook, New York \\ Robert Matthews* \\ *Stony Brook University Hospital \\ HSC Building Level 4, Room 120, SUNY at Stony Brook \\ Stony Brook, NY 11794 \\ Email: robert.matthews@stonybrook.edu
}

Published online Aug. 2, 2019.

DOI: 10.2967/jnumed.119.234658 